\title{
The Boundedness and Exponential Stability Criterions for Nonlinear Hybrid Neutral Stochastic Functional Differential Equations
}

\author{
Xiaofeng Zong, Fuke Wu, and Chengming Huang \\ School of Mathematics and Statistics, Huazhong University of Science and Technology, Wuhan, Hubei 430074, China \\ Correspondence should be addressed to Fuke Wu; wufuke@mail.hust.edu.cn
}

Received 26 April 2013; Accepted 25 June 2013

Academic Editor: Marcia Federson

Copyright (C) 2013 Xiaofeng Zong et al. This is an open access article distributed under the Creative Commons Attribution License, which permits unrestricted use, distribution, and reproduction in any medium, provided the original work is properly cited.

Neutral differential equations have been used to describe the systems that not only depend on the present and past states but also involve derivatives with delays. This paper considers hybrid nonlinear neutral stochastic functional differential equations (HNSFDEs) without the linear growth condition and examines the boundedness and exponential stability. Two illustrative examples are given to show the effectiveness of our theoretical results.

\section{Introduction}

Many dynamic systems not only depend on the present and past states but also involve derivatives with delays. Neutral differential equations have been used to model such systems. Deterministic neutral differential equations were introduced by Hale and Meyer [1] and discussed in Hale and Lunel (see [2]) and Kolmanovskii et al. (for details see also [3, 4]), among others. Such equations were used to study two or more simple oscillatory systems with some interconnections between them, such as Brayton [5], Rubanik [6], and Driver [7].

Generally speaking, many practical systems commonly encounter stochastic perturbations and may experience abrupt changes in their structure and parameters caused by phenomena such as component failures or repairs and abrupt environmental disturbances. Of course, there is no exception to neutral systems, mentioned previous. Taking these stochastic factors into account, Mao and Yuan developed hybrid systems driven by Brownian motion and continuous-time Markovian chain to cope with such a situation (see [8]). Hu et al. [9] investigated the stability and boundedness of stochastic differential delay equations with Markovian switching. Kolmanovskii et al. [10] discussed the neutral stochastic delay differential equations with Markovian switching, also known as hybrid neutral stochastic delay differential equations (HNSDDEs).

The boundedness and stability analysis of the neutral stochastic systems without switching has attracted much attention; see [11-18] to mention a few. For hybrid neutral systems, studying boundedness and stability of the solutions is also a challenging and interesting work. Kolmanovskii et al. [10] established a fundamental theorem of HNSDDEs and discussed the boundedness and exponential stability of the solutions. They also gave an example to show that Markovian can average the subsystems; that is, when some subsystems are stable and others are not stable, the overall system formed by the Markovian switching may be stable. Bao et al. [19] discussed stability in distribution of the HNSDDEs. $\mathrm{Hu}$ and Wang [20] studied the stability in distribution for the general HNSFDEs. The stability of HNSDDEs with interval uncertainty was investigated in [21]. Mao et al. [22] gave a criterion related to almost surely asymptotic stability of HNSDDEs. These results are undoubtedly remarkable.

However, there are few publications on the boundedness and exponential stability of the general HNSFDEs with highly nonlinear terms. To fill in this gap, this work gives the boundedness and exponential stability criterions for such 
HNSFDEs. Moreover, when this HNSFDE degenerates to the HNSDDE, our stability criterions improve the related results in [10]. Further, these stability criterions can also be used to investigate the exponential stability of NSFDEs or NSDDEs with more accurate Lyapunov exponent bound than that obtained in $[23,24]$.

The rest of the paper is arranged as follows. The next section provides necessary notations and definitions for the use of this paper. Section 3 establishes the boundedness and exponential stability criterions of the solutions to HNSFDEs. Section 4 further gives the generalized results for the HNSDDEs with variable time delay. Finally, two illustrative examples are provided to show the effectiveness of our theoretical results.

\section{Notations and Definitions}

Throughout this paper, unless otherwise specified, we use the following notations. $|\cdot|$ denotes both the Euclidean vector norm in $\mathbb{R}^{n}$ and Frobenius matrix norm in $\mathbb{R}^{n \times d}$. If $A$ is a vector or matrix, its transpose is denoted by $A^{T}$. If $A$ is a matrix, its trace norm is denoted by $|A|=$ $\sqrt{\operatorname{trace}\left(A^{T} A\right)}$. Let $(\Omega, \mathfrak{F}, P)$ be a complete probability space with a filtration $\left\{\mathfrak{F}_{t}\right\}_{t \geq 0}$ satisfying the usual conditions; that is, it is right continuous and increasing while $\mathfrak{F}_{0}$ contains all $P$-null sets. Let $w(t)=\left(w_{1}(t), \ldots, w_{d}(t)\right)$ be a $d$-dimensional Brownian motion defined on this probability space. Let $\mathbb{R}_{+}=$ $[0, \infty)$ and $\tau>0$. Denote by $C\left([-\tau, 0], \mathbb{R}^{n}\right)$ the family of continuous functions from $[-\tau, 0]$ to $\mathbb{R}^{n}$ with the norm $\|\varphi\|=$ $\sup _{-\tau \leq \theta \leq 0}|\varphi(\theta)|$. Let $C_{\mathfrak{F}_{0}}^{b}\left([-\tau, 0], \mathbb{R}^{n}\right)$ be the family of all $\mathfrak{F}_{0^{-}}$ measurable bounded $C\left([-\tau, 0], \mathbb{R}^{n}\right)$ valued random variables $\xi=\{\xi(\theta):-\tau \leq \theta \leq 0\} . a \vee b$ represents $\max \{a, b\}$, and $a \wedge b$ denotes $\min \{a, b\}$.

Let $r(t)$ be a Markov chain (independent of $w(t)$ ) taking values in a finite state space $\mathbb{S}=\{1,2, \ldots, m\}$. Assume the generator of $r(t)$ is denoted by $\Gamma=\left(\gamma_{i j}\right)_{m \times m}$, so that

$$
\mathbb{P}\{r(t+\delta)=j \mid r(t)=i\}= \begin{cases}\gamma_{i j} \delta+o(\delta), & \text { if } i \neq j \\ 1+\gamma_{i j} \delta+o(\delta), & \text { if } i=j\end{cases}
$$

where $\delta>0$. Here $\gamma_{i j}$ is the transition rate from $i$ to $j$ and $\gamma_{i j}>$ 0 if $i \neq j$ while $\gamma_{i i}=-\sum_{j \neq i} \gamma_{i j}$. Let us consider the following $n$-dimensional nonlinear HNSFDE:

$$
\begin{aligned}
& d\left[x(t)-u\left(x_{t}, r(t)\right)\right] \\
& \quad=f\left(x_{t}, r(t)\right) d t+g\left(x_{t}, r(t)\right) d w(t), \quad t \geq 0,
\end{aligned}
$$

with initial data $x_{0}=\xi \in C_{\mathfrak{\mho}_{0}}^{b}\left([-\tau, 0] ; \mathbb{R}^{n}\right)$ and $r(0)=r_{0} \in \mathbb{S}$, where

$$
x_{t}=\{x(t+\theta) ;-\tau \leq \theta \leq 0\} \in C\left([-\tau, 0] ; \mathbb{R}^{n}\right),
$$

$u: C\left([-\tau, 0], \mathbb{R}^{n}\right) \times \mathbb{S} \rightarrow \mathbb{R}^{n}, f: C\left([-\tau, 0], \mathbb{R}^{n}\right) \times \mathbb{S} \rightarrow \mathbb{R}^{n}$, and $g: C\left([-\tau, 0], \mathbb{R}^{n}\right) \times \mathbb{S} \rightarrow \mathbb{R}^{n \times d}$. In order to guarantee the existence and uniqueness of the solution to (2), we give the following assumptions for the functionals $u, f$, and $g$.
Assumption 1 (local Lipschitz condition). $f$ and $g$ satisfy the local Lipschitz condition; that is, for each $j>0$ there exists a positive constant $C_{j}$ such that for any maps $\phi, \varphi \in$ $C\left([-\tau, 0] ; \mathbb{R}^{n}\right)$ with $\|\phi\| \vee\|\varphi\| \leq j$

$$
\begin{gathered}
|f(\phi, i)-f(\varphi, i)| \vee|g(\phi, i)-g(\varphi, i)| \\
\leq C_{j}\|\phi-\varphi\|, \quad \forall i \in \mathbb{S},
\end{gathered}
$$

where $\|\phi-\varphi\| \leq \sup _{-\tau \leq \theta \leq 0}|\phi(\theta)-\varphi(\theta)|$.

Assumption 2 (contractive mapping). There exists a positive constant $\kappa \in(0,1)$ such that for all $\phi, \varphi \in C\left([-\tau, 0] ; \mathbb{R}^{n}\right)$ and $i \in \mathbb{S}$

$$
|u(\phi, i)-u(\varphi, i)| \leq \kappa\|\phi-\varphi\|
$$

and $u(0, i)=0$.

Note that the previous assumptions are standard for the existence and uniqueness of the local solutions (see $[19,22])$. Additional conditions should be imposed for the local solution to be global. In view of this, we need a few more notations. Let $C^{2}\left(\mathbb{R}^{n} \times \mathbb{S} ; \mathbb{R}_{+}\right)$denote the family of all nonnegative functions $V(x, i)$ on $\mathbb{R}^{n} \times \mathbb{S}$ which are continuously twice differentiable in $x$. For each $V(x, i) \epsilon$ $C^{2}\left(\mathbb{R}^{n} \times \mathbb{S} ; \mathbb{R}_{+}\right)$, define an operator $\mathscr{L} V$ from $C\left([-\tau, 0] ; \mathbb{R}^{n}\right) \times$ $\mathbb{S}$ to $\mathbb{R}$ :

$$
\begin{aligned}
\mathscr{L} V(\varphi, i) & \\
= & V_{x}(\varphi(0)-u(\varphi, i), i) f(\varphi, i) \\
& +\sum_{j \in \mathbb{S}} \gamma_{i j} V(\varphi(0)-u(\varphi, i), j) \\
& +\frac{1}{2} \operatorname{trace}\left[g^{T}(\varphi, i) V_{x x}(\varphi(0)-u(\varphi, i), i) g(\varphi, i)\right],
\end{aligned}
$$

where

$$
\begin{gathered}
V_{x}(x, i)=\left(\frac{\partial V(x, i)}{\partial x_{1}}, \ldots, \frac{\partial V(x, i)}{\partial x_{n}}\right), \\
V_{x x}(x, i)=\left(\frac{\partial^{2} V(x, i)}{\partial x_{j} \partial x_{l}}\right)_{n \times n} .
\end{gathered}
$$

In the following sections, we will impose the some conditions on the diffusion operator $\mathscr{L} \mathrm{V}$ for the global solution and its asymptotic behavior.

\section{The Boundedness and Exponential Stability of HNSFDEs}

The following theorem gives the boundedness and exponential stability criterions of the solution to (2).

Theorem 3. Let Assumptions 1 and 2 hold. Assume that there are two functions $V \in C^{2}\left(\mathbb{R}^{n} \times \mathbb{S} ; \mathbb{R}_{+}\right), U \in C\left(\mathbb{R}^{n} ; \mathbb{R}_{+}\right)$, three probability measures $\eta, \mu, \bar{\mu}$ on $[-\tau, 0]$, and a number 
of positive constants $\kappa \in(0,1), c, c_{1}, c_{2}, p \geq 1, \lambda_{1}, \lambda_{2}, \lambda_{3}, \lambda_{4}$ such that for any $x \in \mathbb{R}^{n}$ and $(\varphi, i) \in C\left([-\tau, 0], \mathbb{R}_{+}\right) \times \mathbb{S}$

$$
\begin{gathered}
|u(\varphi, i)| \leq \kappa \int_{-\tau}^{0} \varphi(\theta) \eta(d \theta), \\
c_{1}|x|^{p} \leq V(x, i) \leq c_{2}|x|^{p},
\end{gathered}
$$

$\mathscr{L} V(\varphi, i)$

$$
\begin{aligned}
\leq & c-\lambda_{1}|\varphi(0)|^{p}+\lambda_{2} \int_{-\tau}^{0}|\varphi(\theta)|^{p} \mu(d \theta)-\lambda_{3} U(\varphi(0)) \\
& +\lambda_{4} \int_{-\tau}^{0} U(\varphi(\theta)) \bar{\mu}(d \theta) .
\end{aligned}
$$

If $\lambda_{1}>\lambda_{2}$ and $\lambda_{3}>\lambda_{4}$, then we have the following assertions:

(i) for any given initial data $\xi \in C_{\mathfrak{F}_{0}}^{b}\left([-\tau, 0], \mathbb{R}^{n}\right)$, there is a unique global solution $x(t)=x(t ; \xi)$ to the hybrid system (2) on $t \in[-\tau, \infty)$;

(ii) the solution $x(t)$ obeys

$$
\begin{gathered}
\limsup _{t \rightarrow \infty} \mathbb{E}\left|x(t)-u\left(x_{t}, r(t)\right)\right|^{p} \leq \frac{c}{\Lambda}, \\
\limsup _{t \rightarrow \infty} \frac{1}{t} \int_{0}^{t} \mathbb{E} U(x(s)) d s \leq \frac{c}{\lambda_{3}-\lambda_{4}},
\end{gathered}
$$

where $\Lambda:=\bar{\gamma} \wedge(1 / \tau) \log \left(\lambda_{3} / \lambda_{4}\right) \wedge r$ with $\bar{\gamma}$ and $r$ defined by

$$
\begin{aligned}
\bar{\gamma}=\max \{q & >0 ; c_{2} q\left(1+\varepsilon^{1 /(p-1)}\right)^{p-1}-\lambda_{1} \\
+ & {\left.\left[c_{2} q\left(1+\varepsilon^{1 /(p-1)}\right)^{p-1} \frac{\kappa^{p}}{\varepsilon}+\lambda_{2}\right] e^{q \tau}=0, \varepsilon>0\right\} }
\end{aligned}
$$

and $r:=(p / \tau) \log (1 / \kappa)-\ell$ for sufficiently small $\ell>0$.

(iii) If, in addition, $c=0$, then the solution of (2) has properties that

$$
\limsup _{t \rightarrow \infty} \frac{\log \left(\mathbb{E}|x(t)|^{p}\right)}{t} \leq-\Lambda
$$

$$
\begin{aligned}
& \int_{0}^{\infty} \mathbb{E} U(x(s)) d s \\
& \leq \frac{1}{\lambda_{3}-\lambda_{4}}\left[\mathbb{E} V\left(y_{0}, r(0)\right)+\lambda_{2} \int_{-\tau}^{0} \mathbb{E}|x(s)|^{p} d s\right. \\
&\left.+\lambda_{4} \mathbb{E} \int_{-\tau}^{0} U(x(s)) d s\right]
\end{aligned}
$$

where $y_{0}=x(0)-u\left(x_{0}, r(0)\right)$.

Proof. We prove these three assertions, separately. For any given initial data $\xi \in C_{\mathfrak{\mho}_{0}}^{b}\left([-\tau, 0], \mathbb{R}^{n}\right)$, by Assumptions 1 and 2 , there exists a unique maximal local strong solution $x(t)$ to
(2) on $t \in\left[-\tau, \rho_{e}\right)$, where $\rho_{e}$ is the explosion time. To show that this solution is global, we only need to prove that $\rho_{e}=\infty$ a.s. Define $y_{t}=x(t)-u\left(x_{t}, r(t)\right)$, then by Assumption 2, we have

$$
\left|y_{0}\right| \leq|x(0)|+\left|u\left(x_{0}, r(0)\right)\right| \leq(1+\kappa)\|\xi\| .
$$

Let $k_{0}$ be sufficiently lager positive number, such that $\|\xi\|<$ $k_{0}$. For each $k>(1+\kappa) k_{0}$, define the stopping time $\rho_{k}=$ $\inf \left\{t \in\left[0, \rho_{e}\right):\left|y_{t}\right| \geq k\right\}$. Clearly, $\rho_{k}$ is increasing as $k \rightarrow \infty$ and $\rho_{k} \rightarrow \rho_{\infty} \leq \rho_{e}$ a.s. If we can show $\rho_{\infty}=\infty$ a.s., then $\rho_{e}=\infty$, which implies that the solution $x(t)$ is actually global. By the generalized Itô formula (see [20]) and condition (10), we can obtain that, for any $k>k_{0}$ and $t \geq 0$,

$$
\begin{aligned}
\mathbb{E} V\left(y_{\left(t \wedge \rho_{k}\right)}, r\left(t \wedge \rho_{k}\right)\right) \\
=\mathbb{E} V\left(y_{0}, r(0)\right)+\mathbb{E} \int_{0}^{t \wedge \rho_{k}} \mathscr{L} V\left(x_{s}, r(s)\right) d s \\
\leq c t+\mathbb{E} V\left(y_{0}, r(0)\right)-\lambda_{1} \mathbb{E} \int_{0}^{t \wedge \rho_{k}}|x(s)|^{p} d s \\
\quad+\lambda_{2} \mathbb{E} \int_{0}^{t \wedge \rho_{k}} \int_{-\tau}^{0}|x(s+\theta)|^{p} \mu(d \theta) d s \\
\quad-\lambda_{3} \mathbb{E} \int_{0}^{t \wedge \rho_{k}} U(x(s)) d s \\
\quad+\lambda_{4} \mathbb{E} \int_{0}^{t \wedge \rho_{k}} \int_{-\tau}^{0} U(x(s+\theta)) \bar{\mu}(d \theta) d s .
\end{aligned}
$$

By the Fubini theorem, we compute

$$
\begin{aligned}
\mathbb{E} \int_{0}^{t \wedge \rho_{k}} & \int_{-\tau}^{0}|x(s+\theta)|^{p} d \mu(\theta) d s \\
\quad & \mathbb{E} \int_{-\tau}^{0} \int_{0}^{t \wedge \rho_{k}}|x(s+\theta)|^{p} d s d \mu(\theta) \\
\quad \leq & \int_{-\tau}^{0} \mathbb{E}|x(s)|^{p} d s+\mathbb{E} \int_{0}^{t \wedge \rho_{k}}|x(s)|^{p} d s .
\end{aligned}
$$

Similarly,

$$
\begin{aligned}
& \mathbb{E} \int_{0}^{t \wedge \rho_{k}} \int_{-\tau}^{0} U(x(s+\theta)) \bar{\mu}(d \theta) d s \\
& \quad \leq \int_{-\tau}^{0} \mathbb{E} U(x(s)) d s+\mathbb{E} \int_{0}^{t \wedge \rho_{k}} U(x(s)) d s .
\end{aligned}
$$


Substituting (18) and (19) into (17) yields

$$
\begin{aligned}
& \mathbb{E} V\left(y_{\left(t \wedge \rho_{k}\right)}, r\left(t \wedge \rho_{k}\right)\right) \\
& \leq \mathbb{E} V\left(y_{0}, r(0)\right) \\
&+\lambda_{2} \int_{-\tau}^{0} \mathbb{E}|x(s)|^{p} d s+\lambda_{4} \mathbb{E} \int_{-\tau}^{0} U(x(s)) d s \\
&+c t-\left(\lambda_{1}-\lambda_{2}\right) \mathbb{E} \\
& \times \int_{0}^{t \wedge \rho_{k}}|x(s)|^{p} d s-\left(\lambda_{3}-\lambda_{4}\right) \mathbb{E} \int_{0}^{t \wedge \rho_{k}} U(x(s)) d s \\
& \leq H_{0}+c t,
\end{aligned}
$$

where

$$
\begin{aligned}
H_{0}= & \mathbb{E} V\left(y_{0}, r(0)\right)+\lambda_{2} \int_{-\tau}^{0} \mathbb{E}|x(s)|^{p} d s \\
& +\lambda_{4} \mathbb{E} \int_{-\tau}^{0} U(x(s)) d s .
\end{aligned}
$$

Note that

$$
\begin{aligned}
& \mathbb{E} V\left(y_{\left(t \wedge \rho_{k}\right)}, r\left(t \wedge \rho_{k}\right)\right) \\
& \quad \geq \mathbb{E}\left[V\left(y_{\left(t \wedge \rho_{k}\right)}, r\left(t \wedge \rho_{k}\right)\right) 1_{\left\{\rho_{k} \leq t\right\}}\right] \geq c_{1} k^{p} \mathbb{P}\left\{\rho_{k} \leq t\right\} .
\end{aligned}
$$

Hence,

$$
c_{1} k^{p} \mathbb{P}\left\{\rho_{k} \leq t\right\} \leq H_{0}+c t
$$

Then, for any $t>0$,

$$
\lim _{k \rightarrow \infty} \mathbb{P}\left\{\rho_{k} \leq t\right\}=0
$$

which together with the arbitrariness of $t$ implies that $\rho_{\infty}=$ $\infty$ a.s. Therefore, the solution $x(t)$ is global, and assertion (i) follows. $\gamma>0$

Then by Itô's formula and condition (10), we have, for any

$$
\begin{aligned}
& \mathbb{E} e^{\gamma\left(t \wedge \rho_{k}\right)} V\left(y_{\left.\left(t \wedge \rho_{k}\right)\right)}, r\left(t \wedge \rho_{k}\right)\right) \\
& =\mathbb{E} V\left(y_{0}, r(0)\right) \\
& \quad+\mathbb{E} \int_{0}^{t \wedge \rho_{k}} e^{\gamma s}\left[\gamma V\left(y_{s}, r(s)\right)+\mathscr{L} V\left(x_{s}, r(s)\right)\right] d s
\end{aligned}
$$

$$
\begin{aligned}
& \leq \mathbb{E} V\left(y_{0}, r(0)\right)+\gamma \mathbb{E} \int_{0}^{t \wedge \rho_{k}} e^{\gamma s} V\left(y_{s}, r(s)\right) d s \\
& -\lambda_{1} \mathbb{E} \int_{0}^{t \wedge \rho_{k}} e^{\gamma s}|x(s)|^{p} d s \\
& +\lambda_{2} \mathbb{E} \int_{0}^{t \wedge \rho_{k}} \int_{-\tau}^{0} e^{\gamma s}|x(s+\theta)|^{p} \mu(d \theta) d s \\
& +c \int_{0}^{t} e^{\gamma s} d s \\
& -\lambda_{3} \mathbb{E} \int_{0}^{t \wedge \rho_{k}} e^{\gamma s} U(x(s)) d s \\
& +\lambda_{4} \mathbb{E} \int_{0}^{t \wedge \rho_{k}} e^{\gamma s} \int_{-\tau}^{0} U(x(s+\theta)) \bar{\mu}(d \theta) d s .
\end{aligned}
$$

For $p \geq 1$ and any $\varepsilon>0$, we have

$$
\begin{aligned}
V & \left(y_{s}, r(s)\right) \\
& \leq c_{2}\left|x(s)-u\left(x_{s}, r(s)\right)\right|^{p} \\
& \leq c_{2}\left[1+\varepsilon^{1 /(p-1)}\right]^{p-1}\left(|x(s)|^{p}+\frac{1}{\varepsilon}\left|u\left(x_{s}, r(s)\right)\right|^{p}\right) \\
& \leq c_{2}\left[1+\varepsilon^{1 /(p-1)}\right]^{p-1}\left[|x(s)|^{p}+\frac{\kappa^{p}}{\varepsilon} \int_{-\tau}^{0}|x(s+\theta)|^{p} d \eta(\theta)\right],
\end{aligned}
$$

where we used the Hölder inequality and condition (8). Substituting (26) into (25), we obtain

$$
\begin{aligned}
e^{\gamma\left(t \wedge \rho_{k}\right)} & \mathbb{E} V\left(y_{\left(t \wedge \rho_{k}\right)}, r\left(t \wedge \rho_{k}\right)\right) \\
\leq & \mathbb{E} V\left(y_{0}\right)+\left[c_{2} \gamma\left(1+\varepsilon^{1 /(p-1)}\right)^{p-1}-\lambda_{1}\right] \\
& \times \mathbb{E} \int_{0}^{t \wedge \rho_{k}} e^{\gamma s} V(x(s)) d s \\
& +c_{2} \gamma\left(1+\varepsilon^{1 /(p-1)}\right)^{p-1} \frac{\mathcal{\kappa}^{p}}{\varepsilon} \\
& \times \mathbb{E} \int_{0}^{t \wedge \rho_{k}} \int_{-\tau}^{0} e^{\gamma s}|x(s+\theta)|^{p} \eta(d \theta) d s \\
& +\lambda_{2} \mathbb{E} \int_{0}^{t \wedge \rho_{k}} \int_{-\tau}^{0} e^{\gamma s}|x(s+\theta)|^{p} \mu(d \theta) d s+c \int_{0}^{t} e^{\gamma s} d s \\
& -\lambda_{3} \mathbb{E} \int_{0}^{t \wedge \rho_{k}} e^{\gamma s} U(x(s)) d s \\
& +\lambda_{4} \mathbb{E} \int_{0}^{t \wedge \rho_{k}} e^{\gamma s} \int_{-\tau}^{0} U(x(s+\theta)) \bar{\mu}(d \theta) d s .
\end{aligned}
$$

Define a probability measure $v$ on $[-\tau, 0]$

$$
d \nu(\theta)=\frac{c_{2} \gamma\left(1+\varepsilon^{1 /(p-1)}\right)^{p-1}\left(\kappa^{p} / \varepsilon\right) \eta(\theta)+\lambda_{2} \mu(\theta)}{c_{2} \gamma\left(1+\varepsilon^{1 /(p-1)}\right)^{p-1}\left(\kappa^{p / \varepsilon}\right)+\lambda_{2}}
$$


then from (27), we have

$$
\begin{aligned}
\left.\mathbb{E} e^{\gamma\left(t \wedge \rho_{k}\right)} V\left(y_{\left(t \wedge \rho_{k}\right)}\right) r\left(t \wedge \rho_{k}\right)\right) \\
\leq \\
+\left[\mathbb{E} V\left(y_{0}\right)+c \int_{0}^{t} e^{\gamma s} d s\right. \\
+\left[c_{2} \gamma\left(1+\varepsilon^{1 /(p-1)}\right)^{p-1}-\lambda_{1}\right] \\
\quad \times \mathbb{E} \int_{0}^{t \wedge \rho_{k}} e^{\gamma s} V(x(s)) d s \\
+\left[c_{2} \gamma\left(1+\varepsilon^{1 /(p-1)}\right)^{p-1} \frac{\kappa^{p}}{\varepsilon}+\lambda_{2}\right] \\
\quad \times \mathbb{E} \int_{0}^{t \wedge \rho_{k}} e^{\gamma s} \int_{-\tau}^{0}|x(s+\theta)|^{p} \nu(d \theta) d s \\
\quad-\lambda_{3} \mathbb{E} \int_{0}^{t \wedge \rho_{k}} e^{\gamma s} U(x(s)) d s \\
+\lambda_{4} \mathbb{E} \int_{0}^{t \wedge \rho_{k}} e^{\gamma s} \int_{-\tau}^{0} U(x(s+\theta)) \bar{\mu}(d \theta) d s .
\end{aligned}
$$

By the Fubini theorem

$$
\begin{aligned}
& \int_{0}^{t} e^{\gamma s} \int_{-\tau}^{0}|x(s+\theta)|^{p} d \nu(\theta) d s \\
& \quad=\int_{-\tau}^{0} \int_{0}^{t} e^{\gamma(s+\theta)}|x(s+\theta)|^{p} d s d \nu(\theta) \\
& \quad \leq e^{\gamma \tau} \int_{-\tau}^{0} e^{\gamma s}|x(s)|^{p} d s+e^{\gamma \tau} \int_{0}^{t} e^{\gamma s}|x(s)|^{p} d s,
\end{aligned}
$$

we have from (29)

$$
\begin{aligned}
& \mathbb{E} e^{\gamma\left(t \wedge \rho_{k}\right)} V\left(y_{\left(t \wedge \rho_{k}\right)}, r\left(t \wedge \rho_{k}\right)\right) \\
& \leq \mathbb{E} V\left(y_{0}\right)+\left[c_{2} \gamma\left(1+\varepsilon^{1 /(p-1)}\right)^{p-1} \frac{\kappa^{p}}{\varepsilon}+\lambda_{2}\right] \\
& \quad \times e^{\gamma \tau} \int_{-\tau}^{0} e^{\gamma s} \mathbb{E} V(x(s)) d s+c \int_{0}^{t} e^{\gamma s} d s \\
& +\left(c_{2} \gamma\left(1+\varepsilon^{1 /(p-1)}\right)^{p-1}\right. \\
& \left.\quad-\lambda_{1}+\left[c_{2} \gamma\left(1+\varepsilon^{1 /(p-1)}\right)^{p-1} \frac{\kappa^{p}}{\varepsilon}+\lambda_{2}\right] e^{\gamma \tau}\right) \\
& \quad \times \mathbb{E} \int_{0}^{t \wedge \rho_{k}} e^{\gamma s} V(x(s)) d s \\
& +\lambda_{4} e^{\gamma \tau} \int_{-\tau}^{0} e^{\gamma_{s}} \mathbb{E} U(x(s)) d s-\left[\lambda_{3}-\lambda_{4} e^{\gamma \tau}\right] \\
& \quad \times \mathbb{E} \int_{0}^{t \wedge \rho_{k}} e^{\gamma s} U(x(s)) d s .
\end{aligned}
$$

\section{Denote}

$$
\begin{gathered}
l(\gamma)=\lambda_{3}-\lambda_{4} e^{\gamma \tau} \\
h(\gamma, \varepsilon)=c_{2} \gamma\left(1+\varepsilon^{1 /(p-1)}\right)^{p-1} \\
-\lambda_{1}+\left[c_{2} \gamma\left(1+\varepsilon^{1 /(p-1)}\right)^{p-1} \frac{\kappa^{p}}{\varepsilon}+\lambda_{2}\right] e^{\gamma \tau} .
\end{gathered}
$$

Let $\varepsilon$ be fixed; then it is easy to obtain $h_{\gamma}^{\prime}(\gamma, \varepsilon)>0$ and $h(0, \varepsilon)=$ $-\lambda_{1}+\lambda_{2}<0$, which implies that for any fixed $\varepsilon>0$, function $h(\cdot, \varepsilon)$ has a unique positive root, denoted by $q$. Choose a $\varepsilon=$ $\varepsilon^{*}>0$ such that

$$
\bar{\gamma}=\sup _{\varepsilon>0, h(q, \varepsilon)=0} q=\sup _{h\left(q, \varepsilon^{*}\right)=0} q .
$$

Noting that for any $\gamma \in(0, \Lambda], h\left(\gamma, \varepsilon^{*}\right) \leq 0$ and $l(\gamma) \geq 0$, we therefore have

$$
\begin{aligned}
& \mathbb{E} e^{\gamma\left(t \wedge \rho_{k}\right)} V\left(y_{\left(t \wedge \rho_{k}\right)}, r\left(t \wedge \rho_{k}\right)\right) \\
& \leq \mathbb{E} V\left(y_{0}\right)+\lambda_{4} e^{\gamma \tau} \int_{-\tau}^{0} e^{\gamma s} \mathbb{E} U(x(s)) d s+c \int_{0}^{t} e^{\gamma s} d s \\
&+\left[c_{2} \gamma\left(1+\varepsilon^{*(1 /(p-1))}\right)^{p-1} \frac{\kappa^{p}}{\varepsilon^{*}}+\lambda_{2}\right] e^{\gamma \tau} \\
& \times \int_{-\tau}^{0} e^{\gamma s} \mathbb{E} V(\xi(s)) d s \\
& \leq c_{1} C_{0} \sup _{-\tau \leq \theta \leq 0} \mathbb{E}|\xi(\theta)|^{p}+c \frac{e^{\gamma t}}{\gamma}
\end{aligned}
$$

for some positive constant $C_{0}>1$. Letting $k \rightarrow \infty$, we have

$$
e^{\gamma t} \mathbb{E} V\left(y_{t}, r(t)\right) \leq c_{1} C_{0} \sup _{-\tau \leq \theta \leq 0} \mathbb{E}|\xi(\theta)|^{p}+c \frac{e^{\gamma t}}{\gamma},
$$

which implies the desired assertion (11). Assertion (12) can be obtained from (20) by letting $k \rightarrow \infty$. Hence assertion (ii) follows.

Let $c=0$. For any $\epsilon>0$, we have that

$$
\begin{aligned}
|x(s)|^{p} \leq & {\left[1+\epsilon^{1 /(p-1)}\right]^{p-1} } \\
& \times\left[\left|y_{s}\right|^{p}+\frac{\left|u\left(x_{s}, r(s)\right)\right|^{p}}{\epsilon}\right] \\
\leq & {\left[1+\epsilon^{1 /(p-1)}\right]^{p-1} } \\
& \times\left[\left|y_{s}\right|^{p}+\frac{\kappa^{p}}{\epsilon} \int_{-\tau}^{0}|x(s+\theta)|^{p} d \eta(\theta)\right] .
\end{aligned}
$$


By (34) and (36), we have for $t>s>0$

$$
\begin{aligned}
e^{\gamma s} \mathbb{E} \mid & \left.x(s)\right|^{p} \\
\leq & {\left[1+\epsilon^{1 /(p-1)}\right]^{p-1} } \\
& \times\left[e^{\gamma s} \mathbb{E}\left|y_{s}\right|^{p}+\frac{\kappa^{p}}{\epsilon} e^{\gamma s} \int_{-\tau}^{0} \mathbb{E}|x(s+\theta)|^{p} d \eta(\theta)\right] \\
\leq & {\left[1+\epsilon^{1 /(p-1)}\right]^{p-1} } \\
& \times\left[e^{\gamma s} \mathbb{E}\left|y_{s}\right|^{p}+\frac{\kappa^{p}}{\epsilon} e^{\gamma \tau} \sup _{s-\tau \leq \theta \leq s}\left[e^{\gamma \theta} \mathbb{E}|x(\theta)|^{p}\right]\right] \\
\leq & {\left[1+\epsilon^{1 /(p-1)}\right]^{p-1} } \\
& \times\left[C_{0} \sup _{-\tau \leq \theta \leq 0} \mathbb{E}|\xi(\theta)|^{p}+\frac{\kappa^{p}}{\epsilon} e^{\gamma \tau} \sup _{-\tau \leq \theta \leq t}\left[\mathrm{e}^{\gamma \theta} \mathbb{E}|x(\theta)|^{p}\right] .\right.
\end{aligned}
$$

This inequality also holds for all $-\tau \leq s \leq 0$. In view of $\gamma \leq$ $r<(p / \tau) \log (1 / \kappa)$, there exists a positive number $\epsilon_{0}>0$ such that

$$
\bar{a}\left(\epsilon_{0}\right):=\left[1+\epsilon_{0}^{1 /(p-1)}\right]^{p-1} \frac{\kappa^{p}}{\epsilon_{0}} e^{\gamma \tau}=\left[1+\frac{1}{\epsilon_{0}^{1 /(p-1)}}\right]^{p-1} \kappa^{p} e^{\gamma \tau}<1 .
$$

Therefore,

$$
\begin{aligned}
& \sup _{-\tau \leq s \leq t} e^{\gamma s} \mathbb{E}|x(s)|^{p} \\
& \leq\left[1+\epsilon_{0}^{1 /(p-1)}\right]^{p-1} C_{0} \sup _{-\tau \leq \theta \leq 0} \mathbb{E}|\xi(\theta)|^{p} \\
& \quad+\bar{a}\left(\epsilon_{0}\right) \sup _{-\tau \leq s \leq t} e^{\gamma s} \mathbb{E}|x(s)|^{p}
\end{aligned}
$$

which implies

$$
e^{\gamma t} \mathbb{E}|x(t)|^{p} \leq \frac{\left[1+\epsilon_{0}^{1 /(p-1)}\right]^{p-1} C_{0}}{1-\bar{a}\left(\epsilon_{0}\right)} \sup _{-\tau \leq t \leq 0} \mathbb{E}|\xi(t)|^{p} .
$$

Finally, the required inequality (14) follows by taking logarithm and limitation. Inequality (15) can be also obtained from (20) by letting $k \rightarrow \infty$. Hence assertion (iii) follows.

Remark 4. If the Markovian switching vanishes, Theorem 3 is also true and gives the $p$ th moment exponential stability with the decay rate bigger than that in [24, Theorem 2]. Since the decay rate in [24] is the special case of Theorem 3 with $\varepsilon=1$ in (13).

If $\lambda_{3}=\lambda_{4}=0$, then we directly obtain the following corollary.

Corollary 5. Let Assumptions 1 and 2 hold. Assume that there exist a function $V \in C^{2}\left(\mathbb{R}^{n} \times \mathbb{S} ; \mathbb{R}_{+}\right) \times \mathbb{S}$, two probability measures $\eta, \mu$ on $[-\tau, 0]$, and a number of positive constants $\kappa \in(0,1), c_{1}, c_{2}, p \geq 1, \lambda_{1}, \lambda_{2}$ such that for any $x \in \mathbb{R}^{n}$ and $(\varphi, i) \in C\left([-\tau, 0], \mathbb{R}_{+}\right) \times \mathbb{S}$

$$
\begin{gathered}
|u(\varphi, i)| \leq \kappa \int_{-\tau}^{0} \varphi(\theta) \eta(d \theta), \\
c_{1}|x|^{p} \leq V(x, i) \leq c_{2}|x|^{p}, \\
\mathscr{L} V(\varphi, i) \leq-\lambda_{1}|\varphi(0)|^{p}+\lambda_{2} \int_{-\tau}^{0}|\varphi(\theta)|^{p} \mu(d \theta) .
\end{gathered}
$$

If $\lambda_{1}>\lambda_{2}$, then for any given initial data $\xi \in C_{\mathfrak{\mho}_{0}}^{b}\left([-\tau, 0], \mathbb{R}^{n}\right)$, the solution of (2), denoted by $x(t)=x(t ; \xi)$, has property that

$$
\limsup _{t \rightarrow \infty} \frac{\log \left(\mathbb{E}|x(t)|^{p}\right)}{t} \leq-(\bar{\gamma} \wedge r)
$$

where $\bar{\gamma}$ and $r$ satisfy

$$
\begin{aligned}
\bar{\gamma}=\max \{q & >0 ; c_{2} q\left(1+\varepsilon^{1 /(p-1)}\right)^{p-1}-\lambda_{1} \\
& \left.+\left[c_{2} q\left(1+\varepsilon^{1 /(p-1)}\right)^{p-1} \frac{\kappa^{p}}{\varepsilon}+\lambda_{2}\right] e^{q \tau}=0, \varepsilon>0\right\}
\end{aligned}
$$

and $r:=(p / \tau) \log (1 / \kappa)-\ell$ for sufficiently small $\ell>0$.

Although the $p$ th moment exponential stability and almost sure exponential stability of the exact solution do not imply each other in general, under a restrictive condition the $p$ th moment exponential stability implies almost sure exponential stability (cf. [11]). Here, we give the following theorem about the almost sure exponential stability of the exact solution to (2).

Theorem 6. Let $p \geq 1$. Assume that there exists a constant $K>0$ such that

$|f(\varphi, i)|+|g(\varphi, i)| \leq K\|\varphi\|, \quad \forall(\varphi, i) \in C\left([-\tau, 0], \mathbb{R}_{+}\right) \times \mathbb{S}$.

Then (42) implies

$$
\limsup _{t \rightarrow \infty} \frac{1}{t} \log |x(t)| \leq-\frac{\bar{\gamma} \wedge r}{p} \text { a.s. }
$$

In other words, the pth moment exponential stability implies almost sure exponential stability.

Remark 7. One may question that whether the semimartingale technique can be used to obtain the almost sure exponential stability directly. In fact, semimartingale technique may fail, since it may not be true to transfer the almost sure exponential stability from $x(t)-u\left(x_{t}, r(t)\right)$ to $x(t)$. 


\section{The Boundedness and Exponential Stability of HNSDDEs}

In this section, we investigate the exponential stability of the hybrid NSDDE with varying delay

$$
\begin{aligned}
d[x(t) & -N(x(t-\tau(t)), r(t))] \\
= & F(x(t), x(t-\tau(t)), r(t)) d t \\
& +G(x(t), x(t-\tau(t)), r(t)) d w(t),
\end{aligned}
$$

where $\tau(t): \mathbb{R}^{+} \rightarrow[0, \tau]$ is a continuously differentiable function such that

$$
\frac{d \tau(t)}{d t} \leq \bar{\tau}
$$

for some constant $\bar{\tau}<1$, while

$$
\begin{aligned}
& N: \mathbb{R}^{n} \times \mathbb{S} \rightarrow \mathbb{R}^{n}, \\
& F: \mathbb{R}^{n} \times \mathbb{R}^{n} \times \mathbb{S} \rightarrow \mathbb{R}^{n}, \quad G: \mathbb{R}^{n} \times \mathbb{R}^{n} \times \mathbb{S} \rightarrow \mathbb{R}^{n \times d} .
\end{aligned}
$$

For (46), we impose the following assumptions.

Assumption 8 (local Lipschitz condition). $F$ and $G$ satisfy the local Lipschitz condition; that is, for each $j>0$ there exists a positive constant $C_{j}$ such that

$$
\begin{aligned}
& |F(x, y, i)-F(\bar{x}, \bar{y}, i)| \vee|G(x, y, i)-G(\bar{x}, \bar{y}, i)| \\
& \leq C_{j}(|x-\bar{x}|+|y-\bar{y}|)
\end{aligned}
$$

for all $i \in \mathbb{S}$ and $x, y, \bar{x}, \bar{y} \in \mathbb{R}^{n}$ with $|x| \vee|y| \vee|\bar{x}| \vee|\bar{y}| \leq j$.

Assumption 9 (contractive mapping). $\mathrm{N}$ is a contractive mapping; that is, there exists a positive constant $\kappa \in(0,1)$ such that for all $x, y \in \mathbb{R}^{n}$ and $i \in \mathbb{S}$

$$
|N(x, i)-N(y, i)| \leq \kappa|x-y| .
$$

Under the previous two assumptions, HNSDDE (46) admits a unique local solution. We also need more conditions to guarantee that the local solution is actually global. So we introduce an operator $L V$ from $\mathbb{R}^{n} \times \mathbb{R}^{n} \times \mathbb{S}$ to $\mathbb{R}$ by

$$
\begin{aligned}
L V(x, y, i) \\
=V_{x}(x-N(y, i), i) F(x, y, i) \\
\quad+\sum_{j \in \mathbb{S}} \gamma_{i j} V(x-N(y, i), j) \\
\quad+\frac{1}{2} \operatorname{trace}\left[G^{T}(x, y, i) V_{x x}(x-N(y, i), i) G(x, y, i)\right]
\end{aligned}
$$

for each $V(x, i) \in C^{2}\left(\mathbb{R}^{n} \times \mathbb{S} ; \mathbb{R}_{+}\right)$, and we will impose the same conditions on the diffusion operator $L V$ for the global solution and its asymptotic behavior.

Although HNSDDE can be regarded as the special case of HNSFDEs, we still establish the boundedness and exponential stability criterions of the solution for (46) so as to obtain more accurate results.
Theorem 10. Let Assumptions 8 and 9 hold. Assume that there are functions $V \in C^{2}\left(\mathbb{R}^{n} \times \mathbb{S} ; \mathbb{R}_{+}\right), U \in C\left(\mathbb{R}^{n} ; \mathbb{R}_{+}\right)$as well as a number of positive constants $c, c_{1}, c_{2}, p \geq 1, \lambda_{1}, \lambda_{2}, \lambda_{3}, \lambda_{4}$ such that for any $x, y \in \mathbb{R}^{n}$ and $i \in \mathbb{S}$,

$$
\begin{gathered}
c_{1}|x|^{p} \leq V(x, i) \leq c_{2}|x|^{p}, \\
L V(x, y, i) \\
\quad \leq c-\lambda_{1}|x|^{p}+\lambda_{2}|y|^{p}-\lambda_{3} U(x)+\lambda_{4} U(y) .
\end{gathered}
$$

If $\lambda_{1}>\lambda_{2} /(1-\bar{\tau})$ and $\lambda_{3}>\lambda_{4} /(1-\bar{\tau})$, then for any given initial data $\xi \in C_{\mathfrak{F}_{0}}^{b}\left([-\tau, 0], \mathbb{R}^{n}\right),(46)$ admits a unique global solution $x(t)=x(t ; \xi)$. Moreover, we have the following assertions:

(i) the solution $x(t)$ obeys

$$
\limsup _{t \rightarrow \infty} \mathbb{E}|x(t)-N(x(t-\tau(t)), r(t))|^{p} \leq \frac{c}{\Lambda},
$$

$$
\limsup _{t \rightarrow \infty} \frac{1}{t} \int_{0}^{t} \mathbb{E} U(x(s)) d s \leq \frac{c}{\lambda_{3}-\lambda_{4} /(1-\bar{\tau})},
$$

where $\Lambda:=\bar{\gamma} \wedge(1 / \tau) \log \left(\lambda_{3} / \lambda_{4}\right) \wedge r$ with $\bar{\gamma}$ and $r$ defined by

$$
\begin{aligned}
\bar{\gamma}=\max \{q & >0 ; c_{2} q\left(1+\varepsilon^{1 /(p-1)}\right)^{p-1}-\lambda_{1} \\
+ & {\left.\left[c_{2} q\left(1+\varepsilon^{1 /(p-1)}\right)^{p-1} \frac{\kappa^{p}}{\varepsilon}+\lambda_{2}\right] e^{q \tau}=0, \varepsilon>0\right\} }
\end{aligned}
$$

and $r:=(p / \tau) \log (1 / \kappa)-\ell$ for sufficiently small $\ell>0$.

(ii) If, in addition, $c=0$, then the solution to (46) has properties that

$$
\limsup _{t \rightarrow \infty} \frac{\log \left(\mathbb{E}|x(t)|^{p}\right)}{t} \leq-\Lambda
$$

$$
\begin{aligned}
& \int_{0}^{\infty} \mathbb{E} U(x(s)) d s \\
& \leq \frac{1}{\lambda_{3}-\lambda_{4} /(1-\bar{\tau})} \\
& \quad \times[\mathbb{E} V(x(0)-N(x(-\tau(0)), r(0)), r(0)) \\
&\left.\quad+\frac{\lambda_{2}}{1-\bar{\tau}} \int_{-\tau}^{0} \mathbb{E}|x(s)|^{p} d s+\frac{\lambda_{4}}{1-\bar{\tau}} \mathbb{E} \int_{-\tau}^{0} U(x(s)) d s\right] .
\end{aligned}
$$

Proof. The proof is similar to that of Theorem 3, so we only give an outlined one. Denote $y_{t}=x(t)-N(x(t-\tau(t)), r(t))$. Let $\rho_{k}$ be the stopping time defined similarly in the proof 
of Theorem 3. By the generalized Itô formula (see [10]) and condition (53), we can obtain that, for any $k>k_{0}$ and $t \geq 0$,

$$
\begin{aligned}
& \mathbb{E} V\left(y_{\left(t \wedge \rho_{k}\right)}, r\left(t \wedge \rho_{k}\right)\right) \\
& \leq c t+\mathbb{E} V\left(y_{0}, r(0)\right)-\lambda_{1} \mathbb{E} \int_{0}^{t \wedge \rho_{k}}|x(s)|^{p} d s \\
& \quad+\lambda_{2} \mathbb{E} \int_{0}^{t \wedge \rho_{k}}|x(s-\tau(s))|^{p} d s \\
& \quad-\lambda_{3} \mathbb{E} \int_{0}^{t \wedge \rho_{k}} U(x(s)) d s \\
& +\lambda_{4} \mathbb{E} \int_{0}^{t \wedge \rho_{k}} U(x(s-\tau(s))) d s .
\end{aligned}
$$

Noting that

$$
\begin{aligned}
\int_{0}^{t}|x(s-\tau(s))|^{p} d s \\
\leq \frac{1}{1-\bar{\tau}} \int_{-\tau}^{0}|x(s)|^{p} d s \\
\quad+\frac{1}{1-\bar{\tau}} \int_{0}^{t}|x(s)|^{p} d s,
\end{aligned}
$$

then we have

$$
\begin{aligned}
\mathbb{E} V\left(y_{\left(t \wedge \rho_{k}\right)}, r\left(t \wedge \rho_{k}\right)\right) \\
\leq c t+\mathbb{E} V\left(y_{0}, r(0)\right)+\frac{\lambda_{2}}{1-\bar{\tau}} \mathbb{E} \int_{-\tau}^{0}|x(s)|^{p} d s \\
\quad+\frac{\lambda_{4}}{1-\bar{\tau}} \int_{-\tau}^{0} \mathbb{E} U(x(s)) d s \\
\quad-\left(\lambda_{1}-\frac{\lambda_{2}}{1-\bar{\tau}}\right) \mathbb{E} \int_{0}^{t \wedge \rho_{k}}|x(s)|^{p} d s \\
\quad-\left(\lambda_{3}-\frac{\lambda_{4}}{1-\bar{\tau}}\right) \mathbb{E} \int_{0}^{t \wedge \rho_{k}} U(x(s)) d s .
\end{aligned}
$$

Then by the similar arguments used in the proof of Theorem 3, we easily obtain $\rho_{k} \rightarrow \infty$ as $k \rightarrow \infty$; that is, the solution $x(t)$ is global. The desired assertions (55) and (58) follow from (61) by letting $k \rightarrow \infty$.

Applying Itôs formula to $e^{\gamma t} V\left(y_{t}, r(t)\right)$ and using condition (53), we have for any $\gamma \in(0, \Lambda]$

$$
\begin{aligned}
& \mathbb{E} e^{\gamma\left(t \wedge \rho_{k}\right)} V\left(y_{t}, r(t)\right) \\
&=\mathbb{E} V\left(y_{0}, r(0)\right) \\
&+\mathbb{E} \int_{0}^{t \wedge \rho_{k}} e^{\gamma s}\left[\gamma V\left(y_{s}, r(s)\right)\right. \\
&+L V(x(s), x(s-\tau(s)), r(s))] d s
\end{aligned}
$$

$$
\begin{aligned}
\leq & \mathbb{E} V\left(y_{0}, r(0)\right)+\gamma \mathbb{E} \int_{0}^{t \wedge \rho_{k}} e^{\gamma s} V\left(y_{s}, r(s)\right) d s \\
& -\lambda_{1} \mathbb{E} \int_{0}^{t \wedge \rho_{k}} e^{\gamma s}|x(s)|^{p} d s \\
& +\lambda_{2} \mathbb{E} \int_{0}^{t \wedge \rho_{k}} e^{\gamma s}|x(s-\tau(s))|^{p} d s \\
& -\lambda_{3} \mathbb{E} \int_{0}^{t \wedge \rho_{k}} e^{\gamma s} U(x(s)) d s \\
& +\lambda_{4} \mathbb{E} \int_{0}^{t \wedge \rho_{k}} e^{\gamma s} U(x(s-\tau(s))) d s .
\end{aligned}
$$

For $p \geq 1$ and any $\varepsilon>0$, we have

$$
\begin{aligned}
& V\left(y_{s}, r(s)\right) \\
& \leq c_{2}|x(s)-N(x(s-\tau(s)), r(s))|^{p} \\
& \leq c_{2}\left[1+\varepsilon^{1 /(p-1)}\right]^{p-1} \\
& \times\left(|x(s)|^{p}+\frac{1}{\varepsilon}|N(x(s-\tau(s)), r(s))|^{p}\right) \\
& \leq c_{2}\left[1+\varepsilon^{1 /(p-1)}\right]^{p-1} \\
& \times\left[|x(s)|^{p}+\frac{\kappa^{p}}{\varepsilon}|x(s-\tau(s))|^{p}\right],
\end{aligned}
$$

where we used the Hölder inequality and Assumption 9. Substituting (63) into (62), we therefore obtain

$$
\begin{aligned}
&\left.\mathbb{E} e^{\gamma\left(t \wedge \rho_{k}\right)} V\left(y_{\left(t \wedge \rho_{\mathrm{k}}\right)}\right) r\left(t \wedge \rho_{k}\right)\right) \\
& \leq \mathbb{E} V\left(y_{0}\right)+\left[c_{2} \gamma\left(1+\varepsilon^{1 /(p-1)}\right)^{p-1}-\lambda_{1}\right] \\
& \quad \times \mathbb{E} \int_{0}^{t \wedge \rho_{k}} e^{\gamma s} V(x(s)) d s \\
& \quad+c_{2} \gamma\left(1+\varepsilon^{1 /(p-1)}\right)^{p-1} \frac{\kappa^{p}}{\varepsilon} \mathbb{E} \\
& \quad \times \int_{0}^{t \wedge \rho_{k}} e^{\gamma s}|x(s-\tau(s))|^{p} d s \\
&+\lambda_{2} \mathbb{E} \int_{0}^{t \wedge \rho_{k}} e^{\gamma s}|x(s-\tau(s))|^{p} d s \\
& \quad-\lambda_{3} \mathbb{E} \int_{0}^{t \wedge \rho_{k}} e^{\gamma s} U(x(s)) d s \\
&+\lambda_{4} \mathbb{E} \int_{0}^{t \wedge \rho_{k}} e^{\gamma s} U(x(s-\tau(s))) d s .
\end{aligned}
$$


Noting that

$$
\begin{aligned}
& \int_{0}^{t} e^{\gamma s}|x(s-\tau(s))|^{p} d s \\
& \leq e^{\gamma \tau} \int_{0}^{t} e^{\gamma(s-\tau(s))}|x(s-\tau(s))|^{p} d s \\
& \leq \frac{e^{\gamma \tau}}{1-\bar{\tau}} \int_{-\tau}^{0} e^{\gamma s}|x(s)|^{p} d s \\
& +\frac{e^{\gamma \tau}}{1-\bar{\tau}} \int_{0}^{t} e^{\gamma s}|x(s)|^{p} d s,
\end{aligned}
$$

then we have from (64)

$$
\begin{aligned}
& \mathbb{E} e^{\gamma\left(t \wedge \rho_{k}\right)} V\left(y_{\left(t \wedge \rho_{k}\right)}, r\left(t \wedge \rho_{k}\right)\right) \\
& \leq \mathbb{E} V\left(y_{0}\right)+\left[c_{2} \gamma\left(1+\varepsilon^{1 /(p-1)}\right)^{p-1} \frac{\kappa^{p}}{\varepsilon}+\lambda_{2}\right] \\
& \times e^{\gamma \tau} \int_{-\tau}^{0} e^{\gamma s} \mathbb{E} V(x(s)) d s \\
&+h(\gamma, \varepsilon) \mathbb{E} \int_{0}^{t \wedge \rho_{k}} e^{\gamma s} V(x(s)) d s \\
&-l(\gamma) \mathbb{E} \int_{0}^{t \wedge \rho_{k}} e^{\gamma s} U(x(s)) d s \\
&+\lambda_{4} \frac{e^{\gamma \tau}}{1-\bar{\tau}} \int_{-\tau}^{0} e^{\gamma s} \mathbb{E} U(x(s)) d s,
\end{aligned}
$$

where

$$
\begin{gathered}
l(\gamma)=\lambda_{3}-\lambda_{4} \frac{e^{\gamma \tau}}{1-\bar{\tau}}, \\
h(\gamma, \varepsilon)=c_{2} \gamma\left(1+\varepsilon^{1 /(p-1)}\right)^{p-1} \\
-\lambda_{1}+\left[c_{2} \gamma\left(1+\varepsilon^{1 /(p-1)}\right)^{p-1} \frac{\kappa^{p}}{\varepsilon}+\lambda_{2}\right] e^{\gamma \tau} .
\end{gathered}
$$

Let $\varepsilon$ be fixed; then it is easy to obtain $h_{\gamma}^{\prime}(\gamma, \varepsilon)>0$ and $h(0, \varepsilon)=$ $-\lambda_{1}+\lambda_{2}<0$, which implies that for any fixed $\varepsilon>0$ function $h(\cdot, \varepsilon)$ has a unique positive root denoted by $q$. Choose a $\varepsilon=$ $\varepsilon^{*}>0$ such that

$$
\bar{\gamma}=\sup _{\varepsilon>0, h(q, \varepsilon)=0} q=\sup _{h\left(q, \varepsilon^{*}\right)=0} q .
$$

Noting that for any $\gamma \in(0, \Lambda], h\left(\gamma, \varepsilon^{*}\right) \leq 0$ and $l(\gamma) \geq 0$. We therefore have

$$
\begin{aligned}
& \mathbb{E} e^{\gamma\left(t \wedge \rho_{k}\right)} V\left(y_{\left(t \wedge \rho_{k}\right)}, r\left(t \wedge \rho_{k}\right)\right) \\
& \leq \mathbb{E} V\left(y_{0}\right)+\lambda_{4} \frac{e^{\gamma \tau}}{1-\bar{\tau}} \int_{-\tau}^{0} e^{\gamma s} \mathbb{E}|x(s)|^{q} d s \\
&+\left[c_{2} \gamma\left(1+\varepsilon^{*(1 /(p-1))}\right)^{p-1} \frac{\kappa^{p}}{\varepsilon^{*}}+\lambda_{2}\right] \\
& \times e^{\gamma \tau} \int_{-\tau}^{0} e^{\gamma s} \mathbb{E} V(\xi(s)) d s \\
& \leq c_{1} C_{0} \sup _{-\tau \leq \theta \leq 0} \mathbb{E}|\xi(\theta)|^{p}
\end{aligned}
$$

for some positive constant $C_{0}>1$. By the similar skills used in the proof of Theorem 3, we can easily obtain the desired assertions (54) and (57).

If the delay $\tau(t)=\tau$ is a fixed constant, then $\bar{\tau}=0$. Hybrid system (46) becomes the following HNSDDE:

$$
\begin{aligned}
d[x(t)- & N(x(t-\tau), r(t))] \\
= & F(x(t), x(t-\tau), r(t)) d t \\
& +G(x(t), x(t-\tau), r(t)) d w(t) .
\end{aligned}
$$

Resorting to Theorem 10, we have the following corollary.

Corollary 11. Let Assumptions 8 and 9 hold. Assume that there are functions $V \in C^{2}\left(\mathbb{R}^{n} \times \mathbb{S} ; \mathbb{R}_{+}\right), U \in C\left(\mathbb{R}^{n} ; \mathbb{R}_{+}\right)$as well as a number of positive constants $c, c_{1}, c_{2}, p \geq 1, \lambda_{1}, \lambda_{2}, \lambda_{3}, \lambda_{4}$ such that for any $x, y \in \mathbb{R}^{n}$ and $i \in \mathbb{S}$

$$
\begin{aligned}
c_{1}|x|^{p} \leq V(x, i) \leq c_{2}|x|^{p}, & \\
L V(x, y, i) \leq & c-\lambda_{1}|x|^{p} \\
& +\lambda_{2}|y|^{p}-\lambda_{3} U(x)+\lambda_{4} U(y) .
\end{aligned}
$$

If $\lambda_{1}>\lambda_{2}$ and $\lambda_{3}>\lambda_{4}$, then for any given initial data $\xi \in C_{\mathfrak{F}_{0}}^{b}\left([-\tau, 0], \mathbb{R}^{n}\right),(70)$ admits a unique global solution $x(t)$. Moreover, we have the following assertions:

(i) the solution $x(t)$ obeys

$$
\begin{gathered}
\limsup _{t \rightarrow \infty} \mathbb{E}|x(t)-N(x(t-\tau), r(t))|^{p} \leq \frac{c}{\Lambda}, \\
\limsup _{t \rightarrow \infty} \frac{1}{t} \int_{0}^{t} \mathbb{E} U(x(s)) d s \leq \frac{c}{\lambda_{3}-\lambda_{4}},
\end{gathered}
$$

where $\Lambda:=\bar{\gamma} \wedge(1 / \tau) \log \left(\lambda_{3} / \lambda_{4}\right) \wedge r$ with $\bar{\gamma}$ and $r$ defined by

$$
\begin{aligned}
\bar{\gamma}=\max \{q & >0 ; c_{2} q\left(1+\varepsilon^{1 /(p-1)}\right)^{p-1}-\lambda_{1} \\
+ & {\left.\left[c_{2} q\left(1+\varepsilon^{1 /(p-1)}\right)^{p-1} \frac{\kappa^{p}}{\varepsilon}+\lambda_{2}\right] e^{q \tau}=0, \varepsilon>0\right\} }
\end{aligned}
$$

and $r:=(p / \tau) \log (1 / \kappa)-\ell$ for sufficiently small $\ell>0$. 
(ii) If, in addition, $c=0$, then the solution of (70) has properties that

$$
\begin{gathered}
\limsup _{t \rightarrow \infty} \frac{\log \left(\mathbb{E}|x(t)|^{p}\right)}{t} \leq-\Lambda, \\
\int_{0}^{\infty} \mathbb{E} U(x(s)) d s \\
\leq \frac{1}{\lambda_{3}-\lambda_{4}} \\
\times\left[\mathbb{E} V\left(y_{0}, r(0)\right)+\lambda_{2} \int_{-\tau}^{0} \mathbb{E}|x(s)|^{p} d s\right. \\
\left.\quad+\lambda_{4} \mathbb{E} \int_{-\tau}^{0} U(x(s)) d s\right] .
\end{gathered}
$$

Further, if $U(x) \equiv 0$, Corollary 11 implies.

Corollary 12. Let Assumptions 8 and 9 hold. Assume that there is a function $V \in C^{2}\left(\mathbb{R}^{n} \times \mathbb{S} ; \mathbb{R}_{+}\right)$and a number of positive constants $c_{1}, c_{2}, p \geq 1, \lambda_{1}, \lambda_{2}$ such that for any $x, y \in \mathbb{R}^{n}$ and $i \in \mathbb{S}$

$$
\begin{gathered}
c_{1}|x|^{p} \leq V(x, i) \leq c_{2}|x|^{p}, \\
L V(x, y, i) \leq-\lambda_{1}|x|^{p}+\lambda_{2}|y|^{p} .
\end{gathered}
$$

If $\lambda_{1}>\lambda_{2}$, then for any given initial data $\xi \in C_{\mho_{0}}^{b}\left([-\tau, 0], \mathbb{R}^{n}\right)$, (70) admits a unique global solution $x(t)$. Moreover, the solution $x(t)$ obeys

$$
\limsup _{t \rightarrow \infty} \frac{\log \left(\mathbb{E}|x(t)|^{p}\right)}{t} \leq-\Lambda,
$$

where $\Lambda:=\bar{\gamma} \wedge r$ with $\bar{\gamma}$ and $r$ defined by

$$
\begin{aligned}
\bar{\gamma}=\max \{q & >0 ; c_{2} q\left(1+\varepsilon^{1 /(p-1)}\right)^{p-1}-\lambda_{1} \\
+ & {\left.\left[c_{2} q\left(1+\varepsilon^{1 /(p-1)}\right)^{p-1} \frac{\kappa^{p}}{\varepsilon}+\lambda_{2}\right] e^{q \tau}=0, \varepsilon>0\right\} }
\end{aligned}
$$

and $r:=(p / \tau) \log (1 / \kappa)-\ell$ for sufficiently small $\ell>0$.

Remark 13. Corollary 12 improves Theorem 5.2 in [10, Chap. 5, pp. 838]. In [10], Theorem 5.2 states that if the assumptions and conditions in Corollary 12 hold, then

$$
\limsup _{t \rightarrow \infty} \frac{1}{t} \log \left(\mathbb{E}|x(t)|^{p}\right) \leq-\Lambda^{*}
$$

with $\bar{\gamma}^{*}>0$ being the unique root to equation

$$
\bar{\gamma}^{*} c_{2}(1+\kappa)^{p-1}+e^{\bar{\gamma}^{*} \tau}\left[\lambda_{2}+\bar{\gamma}^{*} c_{2} \kappa(1+\kappa)^{p-1}\right]=\lambda_{1} .
$$

It is easy to see $\bar{\gamma} \geq \bar{\gamma}^{*}$. Moreover, $(p / \tau) \log (1 / \kappa)>$ $(1 / 2 \tau) \log (1 / \kappa)$. That means $\Lambda^{*} \leq \Lambda$ for sufficiently small $\ell>0$, where $\Lambda$ is defined in Corollary 12 .

\section{Examples}

In this section, we give an example to illustrate the usefulness and flexibility of the theorems developed previously. Let $w(t)$ be a scalar Brownian motion. Let $r(t)$ be a right-continuous Markov chain value in $\mathbb{S}=\{1,2\}$ with generator

$$
\Gamma=\left(\gamma_{i j}\right)_{2 \times 2}=\left(\begin{array}{cc}
-2 & 2 \\
1 & -1
\end{array}\right)
$$

Assume that $w(t)$ and $r(t)$ are independent.

Example 1. Consider the one-dimensional linear HNSDDEs

$$
\begin{aligned}
& d\left[x(t)-\kappa(r(s)) \int_{-\tau}^{0} x(s+\theta) d \theta\right] \\
& =\left[\mu(r(s)) x(t)-2 x(t)^{3}+\int_{-\tau}^{0} x(t+\theta) d \theta\right] d t \\
& +\left[\sigma(r(s)) \int_{-\tau}^{0}|x(t+\theta)|^{2} d \theta+c\right] d w(t),
\end{aligned}
$$

where $\kappa(1)=1 / 8, \kappa(2)=1 / 4, \mu_{1}=-3, \mu_{2}=-4, \sigma(1)=$ $1 / \sqrt{8}, \sigma(2)=1 / \sqrt{2}$, and $\tau=1$. To find out whether $(83)$ is mean-square exponential stability, we use the Lyapunov function

$$
V(x, i)=q_{i}|x|^{2}
$$

where $q_{1}=1$ and $q_{2}=0.5$. One can show that

$$
\begin{aligned}
& \mathscr{L} V(\varphi, i) \\
& =2 q_{i}\left[\varphi(0)+\kappa(i) \int_{-1}^{0} \varphi(\theta) d \theta\right] \\
& \quad \times\left[\mu(i) \varphi(0)-3 \varphi(0)^{3}+\int_{-1}^{0} \varphi(\theta) d \theta\right] \\
& +\sum_{j=1,2} \gamma_{i j} q_{j}\left|\varphi(0)+\frac{1}{8} \int_{-1}^{0} \varphi(\theta) d \theta\right|^{2} \\
& +\left.\left.2 q_{i} \sigma(i)^{2}\left|\int_{-1}^{0}\right| \varphi(\theta)\right|^{2} d \theta\right|^{2}+2 c^{2} .
\end{aligned}
$$


By the elementary inequalities $a^{\alpha} b^{\beta} \leq(\alpha /(\alpha+\beta)) a^{\alpha+\beta}+$ $(\beta /(\alpha+\beta)) b^{\alpha+\beta}$, we have

$$
\begin{aligned}
\mathscr{L} V(\varphi, 1) & 2\left[-3|\varphi(0)|^{2}-3|\varphi(0)|^{4}\right. \\
\leq & \frac{1}{2}\left(|\varphi(0)|^{2}+\int_{-1}^{0}|\varphi(\theta)|^{2} d \theta\right) \\
& +\frac{3}{8} \frac{1}{2}\left(|\varphi(0)|^{2}+\int_{-1}^{0}|\varphi(\theta)|^{2} d \theta\right) \\
& \left.+\frac{3}{8}\left(\frac{3}{4}|\varphi(0)|^{4}+\frac{1}{4} \int_{-1}^{0}|\varphi(\theta)|^{4} d \theta\right)\right] \\
- & \varphi(0)+\frac{1}{8} \int_{-1}^{0}|\varphi(\theta) d \theta|^{2}+\frac{1}{4} \int_{-1}^{0}|\varphi(\theta)|^{4} d \theta+2 c^{2} \\
\leq 2 c^{2} & -\frac{45}{8}|\varphi(0)|^{2} \\
+ & \frac{97}{64} \int_{-1}^{0}|\varphi(\theta)|^{2} d \theta-\frac{87}{16}|\varphi(0)|^{4}+\frac{7}{16} \int_{-1}^{0}|\varphi(\theta)|^{4} d \theta
\end{aligned}
$$

Similarly,

$$
\begin{aligned}
& \mathscr{L} V(\varphi, 2) \\
& \leq 2 c^{2}-\frac{29}{8}|\varphi(0)|^{2} \\
&+\frac{13}{32} \int_{-1}^{0}|\varphi(\theta)|^{2} d \theta-\frac{39}{16}|\varphi(0)|^{4} \\
&+\frac{23}{16} \int_{-1}^{0}|\varphi(\theta)|^{4} d \theta .
\end{aligned}
$$

Let $\lambda_{1}=29 / 8, \lambda_{2}=97 / 64, \lambda_{3}=39 / 16$, and $\lambda_{4}=23 / 16$. Then we have from (86), and (87) for each $i \in \mathbb{S}$,

$$
\begin{aligned}
& \mathscr{L} V(\varphi, i) \\
& \leq 2 c^{2}-\lambda_{1}|\varphi(0)|^{2} \\
& \quad+\lambda_{2} \int_{-1}^{0}|\varphi(\theta)|^{2} d \theta-\lambda_{3}|\varphi(0)|^{4}+\lambda_{4} \int_{-1}^{0}|\varphi(\theta)|^{4} d \theta .
\end{aligned}
$$

Then one can compute $\Lambda=0.5281$ by Theorem 3 . If $c \neq 0$, then

$$
\begin{gathered}
\limsup _{t \rightarrow \infty} \mathbb{E}\left|x(t)-u\left(x_{t}, r(t)\right)\right|^{2} \leq \frac{2 c^{2}}{0.5281}, \\
\limsup _{t \rightarrow \infty} \frac{1}{t} \int_{0}^{t} \mathbb{E}|x(s)|^{4} d s \leq \frac{2 c^{2}}{\lambda_{3}-\lambda_{4}} .
\end{gathered}
$$

If $c=0$, the solution to HNSDDE (83) has the property

$$
\limsup _{t \rightarrow \infty} \frac{\log \left(\mathbb{E}|x(t)|^{2}\right)}{t} \leq-0.5281 .
$$

Example 2. Consider the HNSDDE:

$$
\begin{aligned}
d[x(t) & -N(x(t-\tau), r(t))] \\
= & F(x(t), x(t-\tau), r(t)) d t \\
& +G(x(t), x(t-\tau), r(t)) d w(t),
\end{aligned}
$$

where the functions $N(y, 1)=1 / 3 y, N(y, 2)=1 / 4 y$, $F(x, y, 1)=-2 x-3 x^{3}+y / 4, F(x, y, 2)=-3 x-4 x^{3}+y / 2$, $G(x, y, 1)=1 / 2 y^{2}$, and $G(x, y, 2)=y / 2$. Let $V(x, i)=q_{i}|x|^{2}$ for $q_{1}=1, q_{2}=0.5$. Then one can compute

$$
\begin{aligned}
& L V(x, y, 1) \leq-\frac{41}{2} x^{2}+\frac{23}{24} y^{2}-\frac{21}{4} x^{4}+\frac{1}{6} y^{4}, \\
& L V(x, y, 2) \leq-\frac{29}{16} x^{2}+\frac{31}{64} y^{2}-\frac{13}{4} x^{4}+\frac{1}{4} y^{4} .
\end{aligned}
$$

Then we have from (92) that for $i \in \mathbb{S}$

$$
L V(x, y, i) \leq-\frac{29}{16} x^{2}+\frac{23}{24} y^{2}-\frac{13}{4} x^{4}+\frac{1}{4} y^{4}
$$

One can compute $\Lambda=0.2767$ by Corollary 11 . Then the solution to HNSDDE (91) has the property

$$
\limsup _{t \rightarrow \infty} \frac{\log \left(\mathbb{E}|x(t)|^{2}\right)}{t} \leq-0.2767 \text {. }
$$

\section{Acknowledgments}

The research of Xiaofeng Zong was supported in part by the National Science Foundation of China (Grant no. 11001091). The research of Fuke $\mathrm{Wu}$ was supported in part by the National Science Foundation of China (Grant no. 11001091) and the Program for New Century Excellent Talents in University. The research of Chengming Huang was supported in part by the National Science Foundation of China (Grant no. 91130003) and the Fundamental Research Funds for the Central Universities (Grant no. 2013TS137).

\section{References}

[1] J. K. Hale and K. R. Meyer, A Class of Functional Equations of Neutral Type, Memoirs of the American Mathematical Society, no. 76, American Mathematical Society, Providence, RI, USA, 1967.

[2] J. K. Hale and S. M. V. Lunel, Introduction to Functional Differential Equations, Springer, Berlin, Germany, 1991.

[3] V. B. Kolmanovskii and A. Myshkis, Applied Theory of Functional Differential Equations, Kluwer Academic Publishers, Norwell, Mass, USA, 1992.

[4] V. B. Kolmanovskiı̌ and V. R. Nosov, Stability of FunctionalDifferential Equations, vol. 180 of Mathematics in Science and Engineering, Academic Press, London, UK, 1986.

[5] R. Brayton, "Nonlinear oscillations in a distributed network," Quarterly of Applied Mathematics, vol. 24, pp. 289-301, 1976.

[6] V. P. Rubanik, Oscillations of Quasilinear Systems with Retardation, Nauk, Moscow, Russia, 1969. 
[7] R. D. Driver, "A functional-differential system of neutral type arising in a two-body problem of classical electrodynamics," in Nonlinear Differential Equations and Nonlinear Mechanics, pp. 474-484, Academic Press, New York, NY, USA, 1963.

[8] X. Mao and C. Yuan, Stochastic Differential Equations with Markovian Switching, Imperial College Press, London, UK, 2006.

[9] L. Hu, X. Mao, and Y. Shen, "Stability and boundedness of nonlinear hybrid stochastic differential delay equations," Systems \& Control Letters, vol. 62, no. 2, pp. 178-187, 2013.

[10] V. Kolmanovskii, N. Koroleva, T. Maizenberg, X. Mao, and A. Matasov, "Neutral stochastic differential delay equations with Markovian switching," Stochastic Analysis and Applications, vol. 21, no. 4, pp. 819-847, 2003.

[11] X. Mao, Stochastic Differential Equations and Applications, Horwood, Chichester, UK, 2007.

[12] S. Janković and M. Jovanović, "The pth moment exponential stability of neutral stochastic functional differential equations," Filomat, no. 20, part 1, pp. 59-72, 2006.

[13] S. Janković, J. Randjelović, and M. Jovanović, "Razumikhintype exponential stability criteria of neutral stochastic functional differential equations," Journal of Mathematical Analysis and Applications, vol. 355, no. 2, pp. 811-820, 2009.

[14] X. Mao, "Exponential stability in mean square of neutral stochastic differential-functional equations," Systems \& Control Letters, vol. 26, no. 4, pp. 245-251, 1995.

[15] X. Mao, "Razumikhin-type theorems on exponential stability of neutral stochastic functional-differential equations," SIAM Journal on Mathematical Analysis, vol. 28, no. 2, pp. 389-401, 1997.

[16] X. Mao, "Razumikhin-type theorems on exponential stability of neutral stochastic functional-differential equations," SIAM Journal on Mathematical Analysis, vol. 28, no. 2, pp. 389-401, 1997.

[17] J. Randjelović and S. Janković, "On the pth moment exponential stability criteria of neutral stochastic functional differential equations," Journal of Mathematical Analysis and Applications, vol. 326, no. 1, pp. 266-280, 2007.

[18] F. Wu, S. Hu, and X. Mao, "Razumikhin-type theorem for neutral stochastic functional differential equations with unbounded delay," Acta Mathematica Scientia. Series B. English Edition, vol. 31, no. 4, pp. 1245-1258, 2011.

[19] J. Bao, Z. Hou, and C. Yuan, "Stability in distribution of neutral stochastic differential delay equations with Markovian switching," Statistics \& Probability Letters, vol. 79, no. 15, pp. 1663-1673, 2009.

[20] G. Hu and K. Wang, "Stability in distribution of neutral stochastic functional differential equations with Markovian switching," Journal of Mathematical Analysis and Applications, vol. 385, no. 2, pp. 757-769, 2012.

[21] R. Ravi Kumar and K. T. Chong, "The stability of distributed neutral delay differential systems with Markovian switching," Chaos, Solitons \& Fractals, vol. 45, no. 4, pp. 416-425, 2012.

[22] X. Mao, Y. Shen, and C. Yuan, "Almost surely asymptotic stability of neutral stochastic differential delay equations with Markovian switching," Stochastic Processes and Their Applications, vol. 118, no. 8, pp. 1385-1406, 2008.

[23] Q. Luo, X. Mao, and Y. Shen, "New criteria on exponential stability of neutral stochastic differential delay equations," Systems \& Control Letters, vol. 55, no. 10, pp. 826-834, 2006.
[24] Y. Song and Y. Shen, "New criteria on asymptotic behavior of neutral stochastic functional differential equations," Automatica, vol. 49, no. 2, pp. 626-632, 2013. 


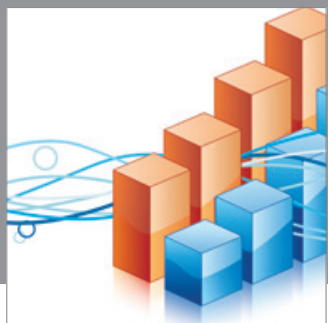

Advances in

Operations Research

mansans

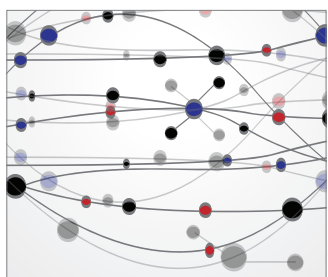

The Scientific World Journal
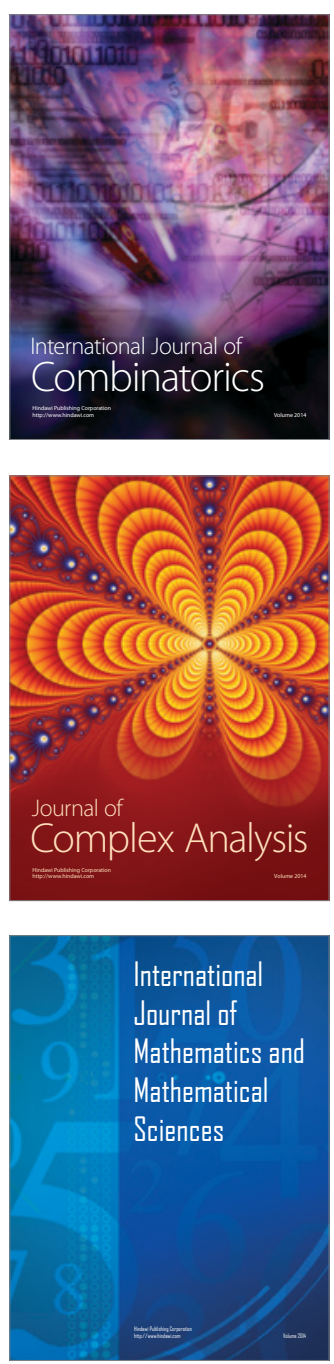
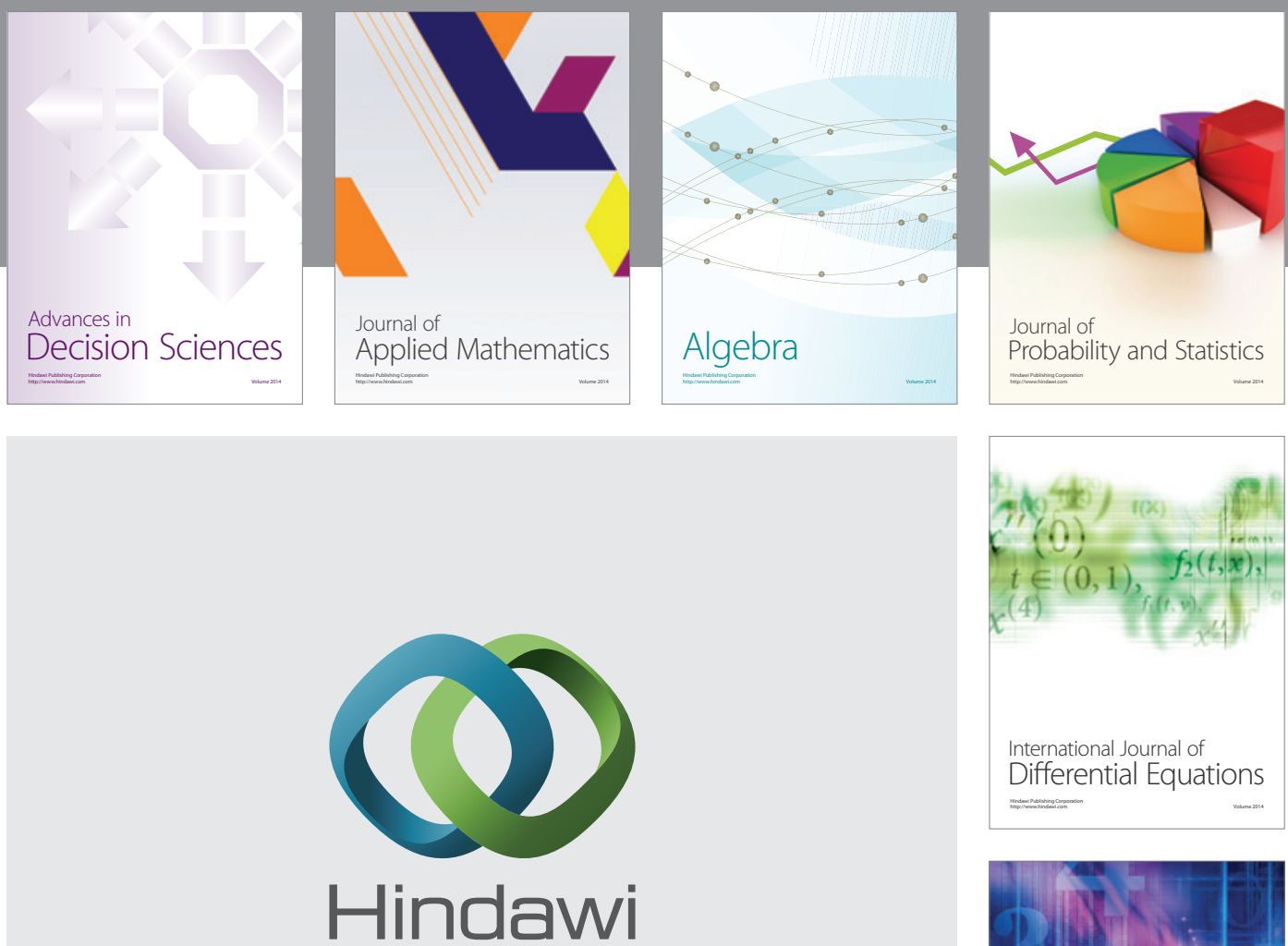

Submit your manuscripts at http://www.hindawi.com
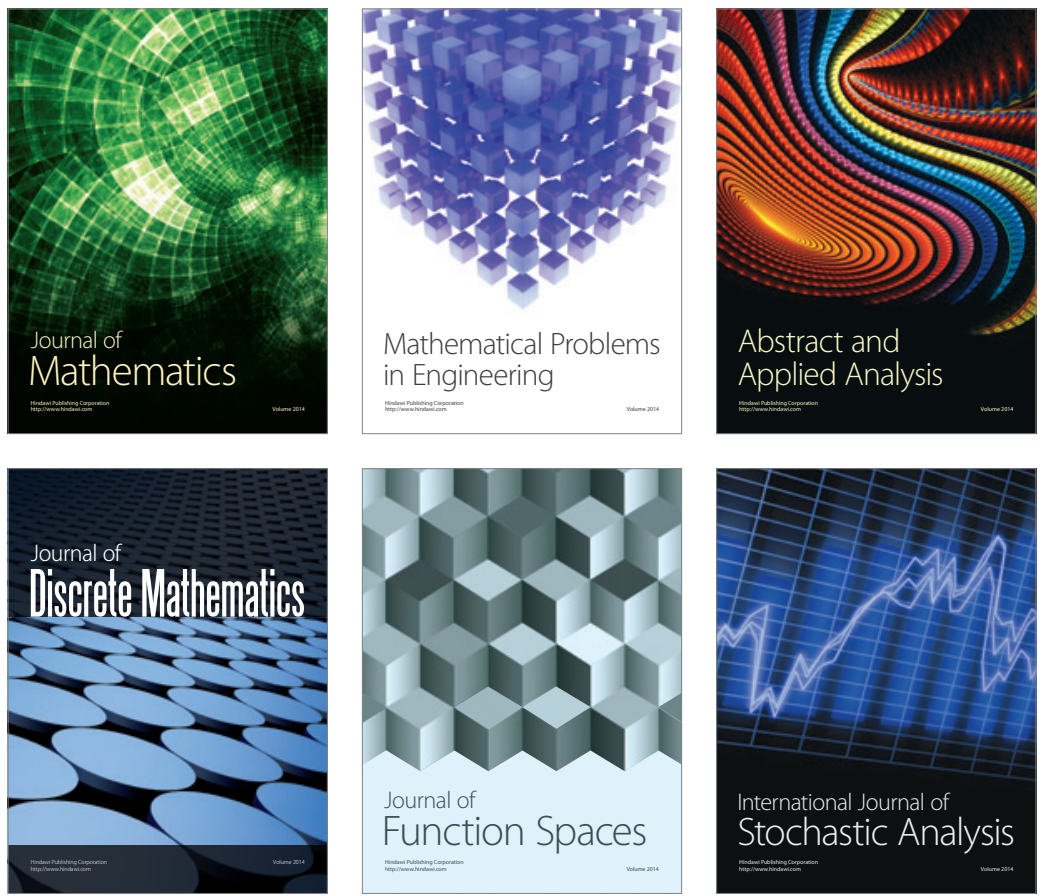

Journal of

Function Spaces

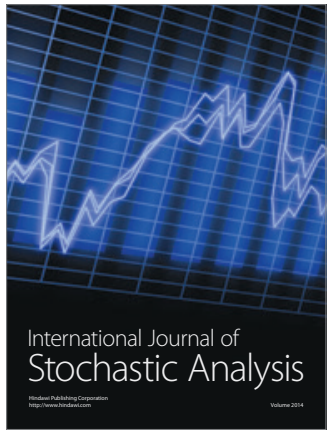

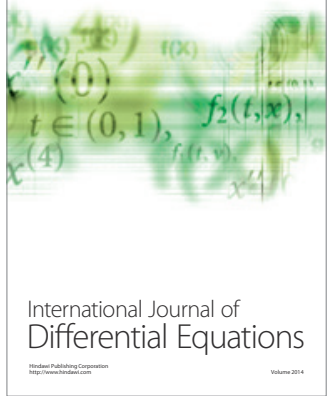
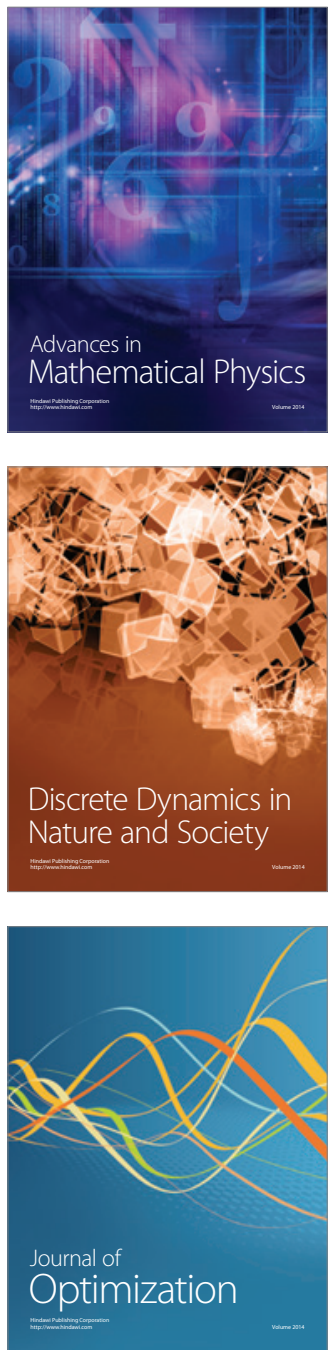